\title{
Arduino based smart home automation
}

\author{
Asish Kumar Majhi", Sujata Dash and Chandan Kumar Barik \\ Department of Computer Application North Orissa University, Baripada, India
}

Received: 15-February-2021; Revised: 10-April-2021; Accepted: 16-April-2021

(C)2021 Asish Kumar Majhi et al. This is an open access article distributed under the Creative Commons Attribution (CC BY) License, which permits unrestricted use, distribution, and reproduction in any medium, provided the original work is properly cited.

\begin{abstract}
Home Automation System has gained popularity due to the advancement of communication technology. A smart home is one of the Internet of Things (IoT) applications that aim to provide an efficient, low-cost automated energy management system for houses that controls the home appliances over the Internet. This paper proposes a low-cost GSM-based automation system for Smart Home to monitor the home appliances remotely. In this paper, an Arduino UNO microcontroller provided with a GSM module is utilized to build the automation system. It provides a cost-effective solution for energy management in the household. It also provides features to cater to natural hazards like fire. It employs several sensors to monitor temperature (LM35), light (LDR Sensor), and fire (Flame Sensor). A relay board is connected to the system which controls the appliances. The proposed home automation system can effectively and efficiently control the electrical appliances via the GSM module and the application code in C developed by Arduino IDE and the application code that are developed in Arduino IDE which uses $C$ programming for coding.
\end{abstract}

\section{Keywords}

Arduino UNO, LDR sensor, Flame sensor, Temperature sensor, Relay module, GSM module.

\section{Introduction}

Now a days as the population increases the needs of human being also increases, hence enhances the consumption of power. But we waste the power unnecessarily for which future generations will suffer. So, if we use different types of sensors according to our needs and automate the system then it is possible to control the usage of power and resources available. This will help to optimize the resources and energy consumption which is very precious in the current situation. Also, this sensor technology can provide a viable solution for patients with restricted mobility [1].

The explained system will work according to the inputs received by the sensors and it can be implemented in Railway platforms. Instead of employing a person to operate the lights and fans in the platforms, it can be automated by using a sensorbased smart system which can ultimately reduce the power consumption. The light sensor only can be used for street lights on the streets by avoiding special cabling for street lights.

*Author for correspondence
Some systems work locally which means the device must be connected to Bluetooth or ZigBee [2-5] to control the automating gadgets. Another method is remotely connecting [2], where the devices are connected to the internet. But if we use the system locally there will be range issue $[3,4]$ as the devices connected to control over Bluetooth can work in a very short range and has IR which is bad for health. And in the case of remotely connected systems, there will be a chance of network failure due to which the system will not work but in our proposal, there will be no disturbance as it is sensing the inputs through sensors and working according to that input.

In this paper, a partial home automation system is developed by assembling a light-dependent resistor (LDR) sensor, temperature sensor, and flame sensor in an Arduino UNO device that receives the data which is sensed by the sensors and processes it through the Arduino and sends the output to respective devices [5]. There are many papers about smart homes but fire detection is not there as a result of the fire will be there it will be a great loss to avoid the situation it is included in the proposed system. If there will be a fire in the house then we will get to know about it by SMS through GSM Module [6] and the SMS will be delivered to the registered user at a five-minute interval until the fire stops. 


\section{IoT technology in home}

IoT is a system that is related between computing devices, digital devices, and other objects which provides unique identifiers (UIDs) and reduces the human effort through data transfer within the network without any direct interaction between human and human or human and computer. The system can connect among a group of devices either be wired or wireless mode. An ideal IoT based device must-have objects like sensors, internet connection, and memory for data storage.

The IoT system is widely accepted all over the world in various sectors which improved efficiency as well as building a smart society. To satisfy the human needs now a days inside a home different sensors and devices are deployed as per the requirement for automation [7]. Hence, a smart home appliance for the future can be addressed through IoT.

\section{Methodology}

In this proposed system the light-dependent resistor (LDR), temperature, and flame sensors are used, a relay is used to combine the system with electrical devices. The relay module is like a middle man which connects the desired circuit (high voltage) with the Arduino (low voltage). Combining all the sensors with
Arduino forms a microcontroller for home automation. Different sensors sense the input and send it to the Arduino to process the input and provide outputs through output devices. The Arduino already contains a program that is coded by a coder. The LDR sensor senses the intensity of light and provides input to Arduino accordingly if decrease in light level electric bulb connected to the board through the relay will turn on and when the intensity of light will increase the bulb will turn off. The temperature sensor will sense the room temperature and when the temperature of the room exceeds than set temperature fan will start and when the temperature decreases to the set temperature fan will stop. The flame sensor continuously senses if there is any fire inside the house, if it detects the fire an SMS will soon be sent to the registered mobile number of the user from the GSM module and will repeat after every five minutes and the buzzer will constantly beep until the fire stops. All these state changes are informed with the help of a beep sound through the buzzer.

The flow diagram (Figure 1) displays the flow of the inputs that are sensed through the sensor and works as the input for the system according to the input the output is processed and the action takes place.

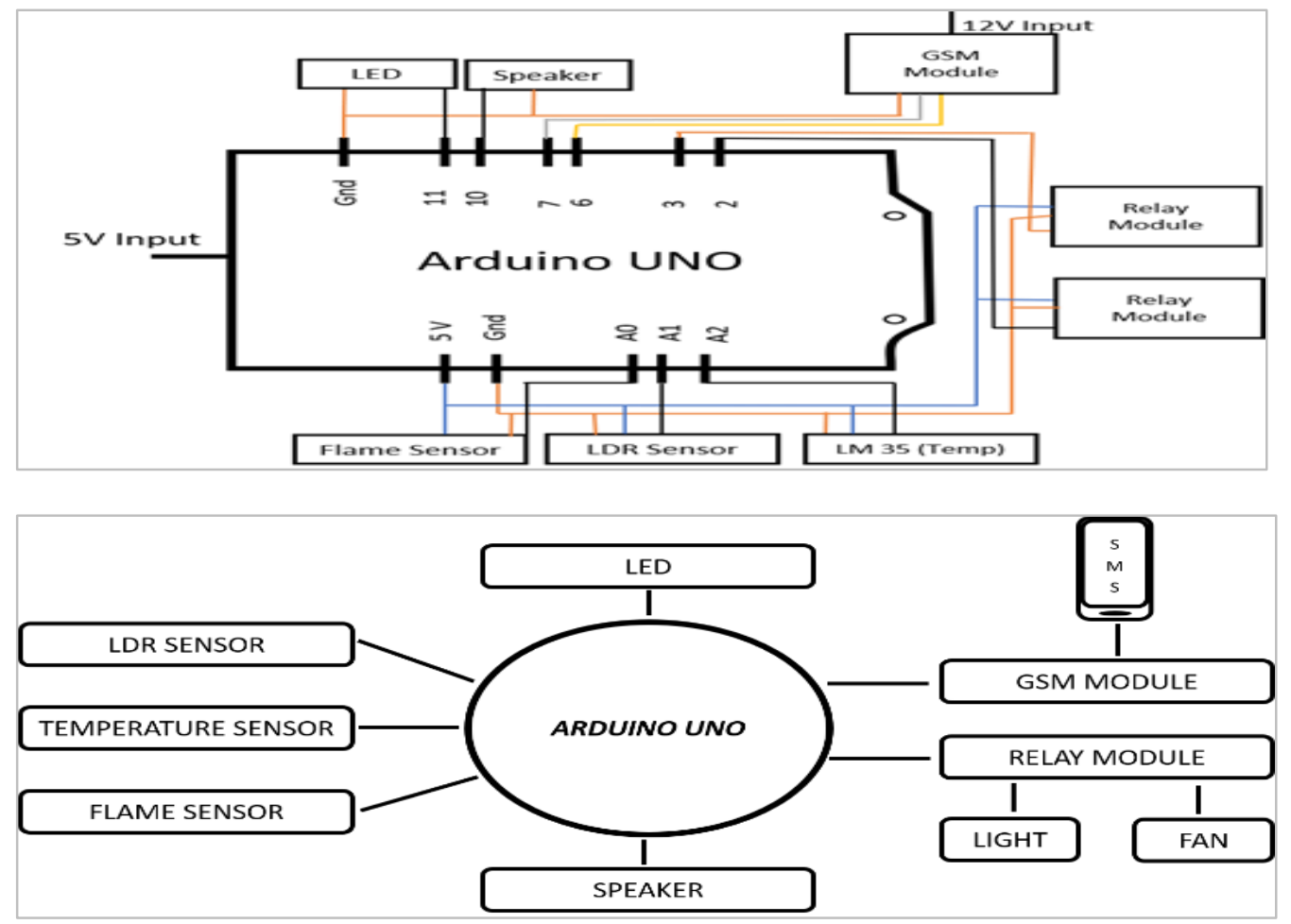

Figure 1 Working principle 


\section{Description of components Arduino UNO}

It is open-source hardware or software which is used for projects. It is licensed under GNU's license so everyone can use it. Arduino board is of different types but here we have used Arduino UNO. The Arduino Uno is an important part of the ATmega328P microcontroller. It has a set of digital along with analog input/output pins. There are 14 digital I/O pins and 6 analog I/O pins on the board. Out of 14 digital I/O pins 6 are capable of PWM output. The setup is programmable with the help of Arduino IDE (Integrated Development Environment) through a type B USB cable. The acceptable voltage range of the system is between 7-20 V and can be supplemented through a USB cable.

Further, the microchip ATmega328 has a programmed boot loader that accepts uploading of new codes of utility without any help of hardware programmers externally. All the components and modules are discussed and presented through Figure 2-Figure 11.

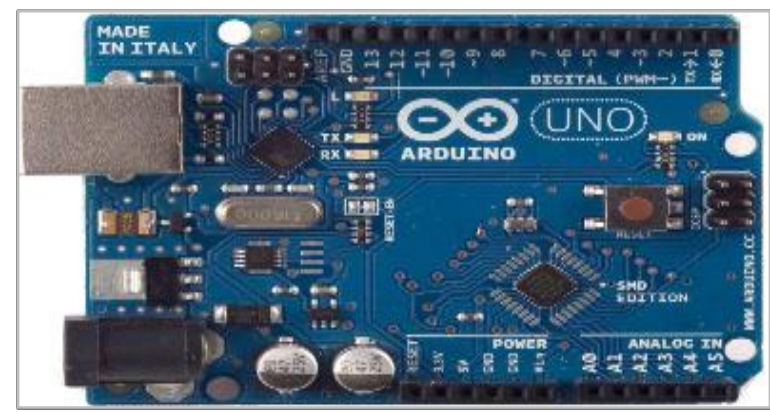

Figure 2 Arduino UNO

\section{GSM module}

GSM or Global System for Mobile Communication works as a communication modem that transmits voice and data services by digital cellular technology. It uses a SIM card to work. It contains a light that blinks after a successful network connection. The frequency bands for the module are $850 \mathrm{MHz}, 900 \mathrm{MHz}, 1800 \mathrm{MHz}$, and $1900 \mathrm{MHz}$ those can carry data at a rate from $64 \mathrm{kbps}$ to $120 \mathrm{Mbps}$.

The GSM module can be connected to a PCB i.e., Printed Circuit Board with different outputs connecting Arduino, 8085, and microcontrollers. The RS232 output is to interface directly with a PC (Personal Computer). The mic and speaker can also be connected to the board through connective pins.

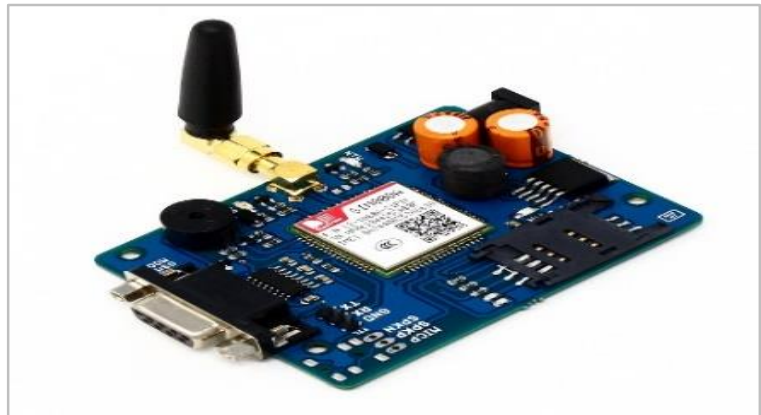

Figure 3 GSM module

\section{Jumper wires}

Jumper wires are the wires that help us to connect different modules and sensors with Arduino. These wires have pins at both ends so we can connect it directly without any soldering. These wires are of three types depending upon their pins at the end i.e.

-Male to Male

-Male to Female

-Female to Female

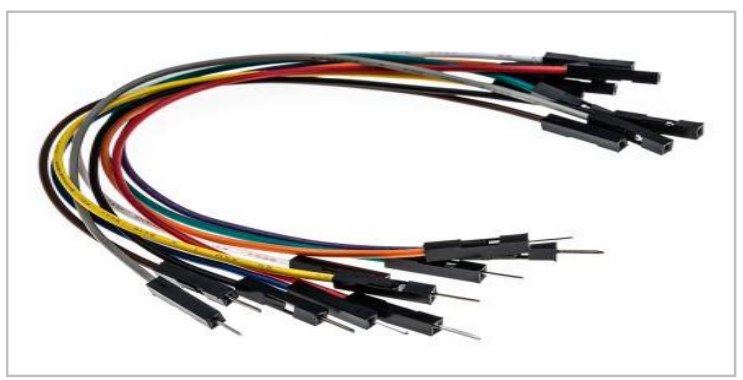

Figure 4 Jumper wires

\section{Breadboard}

A breadboard is a base that helps us to connect electronic devices for prototyping. Using this board, we can provide power to different devices. It is a solderless device so it can be reused whenever required. It contains holes for connecting pins. It has a fixed manner of connection.

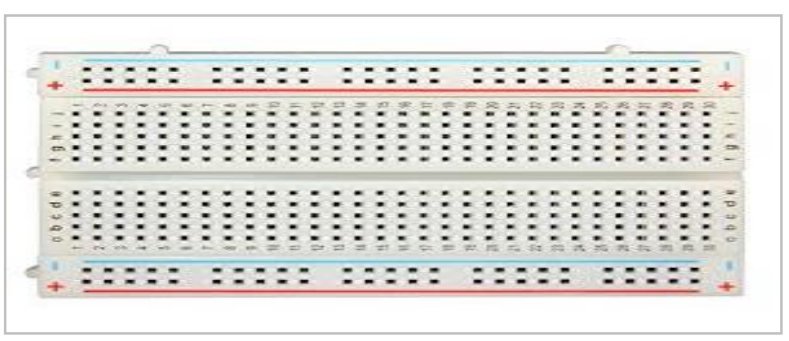

Figure 5 Breadboard 


\section{Relay}

Relay is a module which is like a switch which is used to create a bridge between low voltage (Arduino) and high voltage (AC) devices. It is used in breaking and connecting to a circuit and works as an electrical switch. It allows low voltage connectivity i.e., 5V provided by Arduino pins. There may be one, two, four, or eight channels in the relay module.

If the sensor senses a decrease in intensity of light or temperature increases inside the house, it switches the relay circuit and turns on the electric bulb or fan. Similarly, when the intensity of light increases or the temperature decreases, it switches the relay circuit to turns OFF the electric bulb or fan.

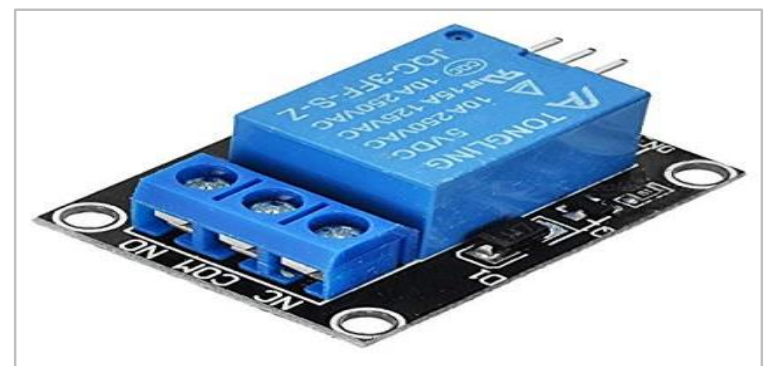

Figure 6 Relay

\section{LDR sensor}

LDR Sensor is known as a light-dependent Resistor sensor. The sensor acts with the environment and gives us the intensity of light available as input and accordingly if the light level decreases, then it sends information to turn on the light and when it senses the light level increases then sends information to turn off the light.

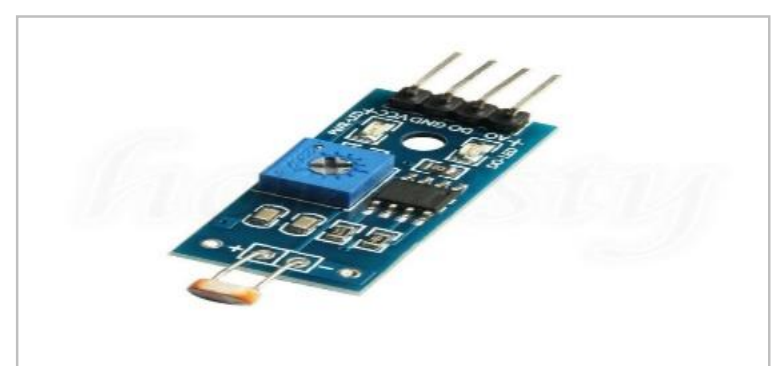

Figure 7 LDR sensor

\section{Temperature sensor}

A temperature sensor is a sensor which is used to sense the temperature inside a provided area or range and sends input and accordingly if the temperature increases then it sends information to turn on the fan to maintain temperature and when it senses the temperature decreases than sends information to turn off the fan.

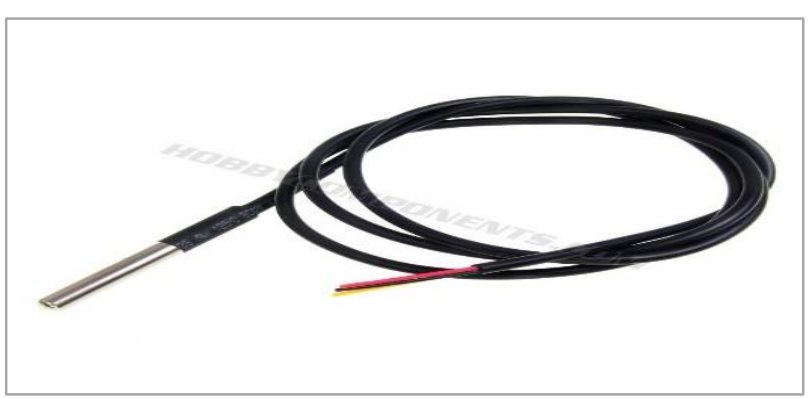

Figure 8 Temperature sensor

Flame sensor

A flame sensor is a sensor that indicates if there is any fire in the region. If there will be any fire breakup then it sends the input and accordingly SMS to the mobile delivered and the buzzer starts beeping continuously.

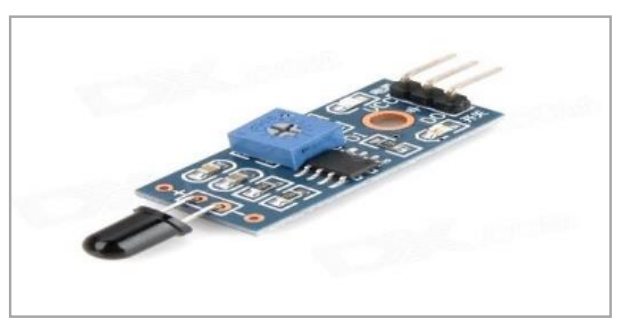

Figure 9 Flame sensor

\section{LED}

LED stands for Light Emitting Diode. It is used to display different outputs. Here it is used to inform about the change in the state of conditions according to the input provided by the sensor. It is connected with a resistor to not get fused.

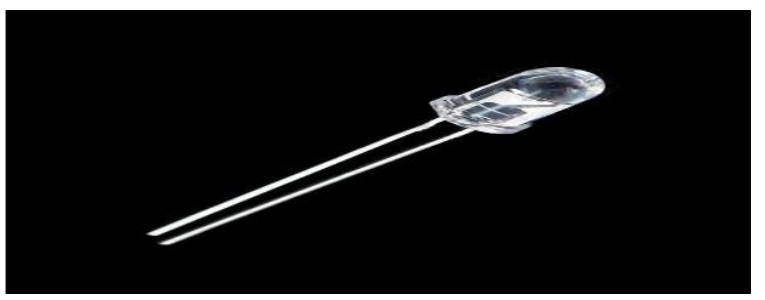

Figure 10 LED

\section{Speaker or buzzer}

A speaker or buzzer is a device which helps us to provide the sound as output. it is connected with the system to provide us the information about the change in the state according to the input received by the sensors. Here it is used as only beeping with the 
change of state but in case of fire breakup, it constantly starts to beep until the fire is extinguished.

Figure 12 is the proposed model without the connection of relay and electric circuit. Here only the Arduino and the sensors were configured with LED and buzzer for output presentation. When there is fire the buzzer starts to beep as the flame sensor connected senses the fire. In this picture, the model is only tested as if it is working or not. In the next picture, the relay is included to show the real-world implementation of the automation system.

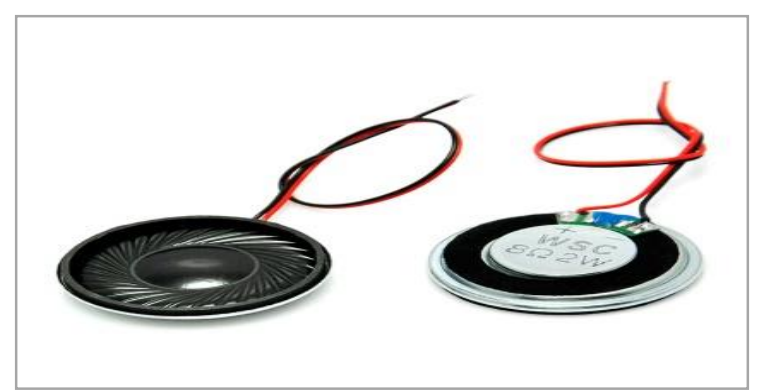

Figure 11 Speaker

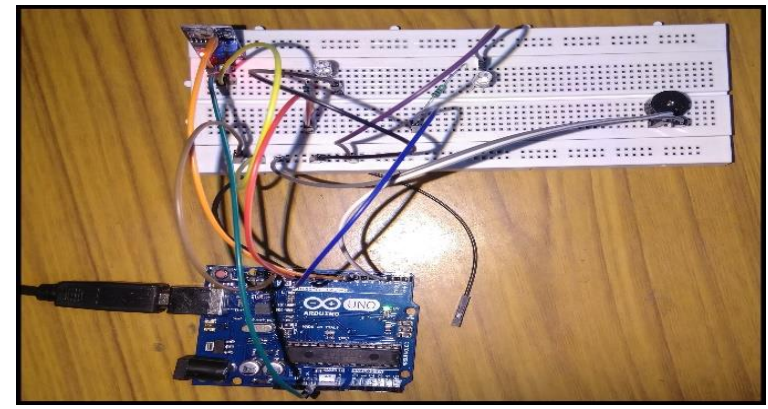

Figure 12 Model without relay and electric circuit

Figure 13 is the proposed model with the connection of relay between the Arduino and the electric circuit i.e., electric bulb. Here for display purpose, we used only electric bulb, here the light intensity is very low so the electric bulb is on but when there is sufficient amount of light the electric bulb will turn off and the process repeats again and again when the inputs change according to different condition i.e., in day time the LDR sensor senses the light amount is high so the light remains off but from evening to morning darkness is there so the light level decreases as a result light turns on.

Figure 14 is the situation where temperature is below 30 degrees so the fan is off but Figure 15 represents the temperature above 30 degree, as a result, the fan started to rotate due to which the room temperature can remain stable. Here we displayed sample for fan by connecting to battery but we can connect it to a wall fan or stand fan through relay like the electric bulb is done.

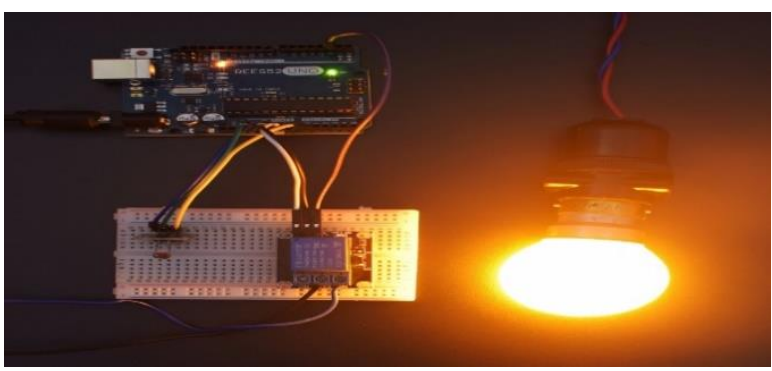

Figure 13 Model with relay and electric circuit

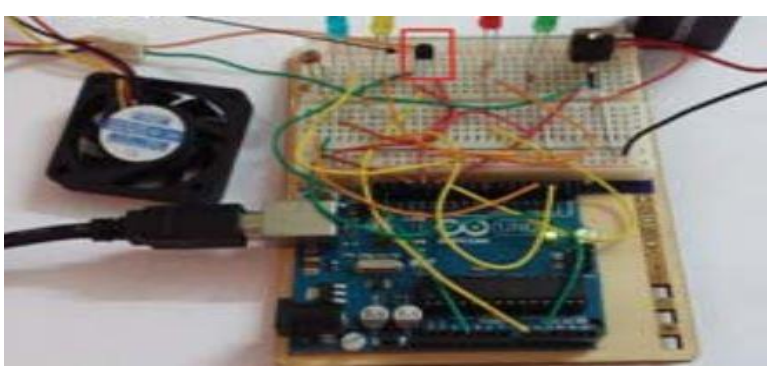

Figure 14 Situation with temperature

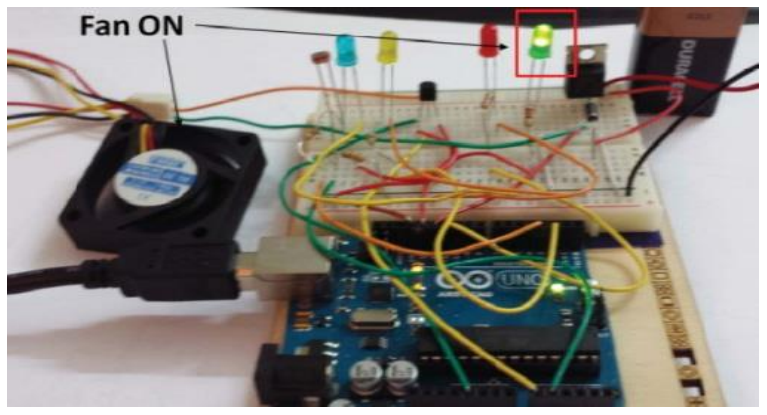

Figure 15 Fan on

\section{Discussion}

This proposed system is designed to reduce human effort and specially get benefited to the bed-ridden patients. The system can be integrated in many places like street light, Railway stations and other public places. The system is neither WIFI nor Bluetooth enabled so it can't be connected with IoT enabled hence it is an automated model for local use only.

\section{Conclusion}

This proposed system is designed and implemented at a very low cost to automate the system using Arduino. It controls all the electronic devices such as electric bulbs, fans, and also alerts if there is any fire breakup. 
The sensor can sense the intensity of light, temperature, and fire. The buzzer will beep when there will be change detected inside the house. When the fire is detected inside the house then there will be a constant beeping of sound as well as the SMS will be sent to the registered mobile number after every five minutes until the fire is extinguished inside the house. The smart system works smoothly and intelligently and effectively to reduce the use of human effort and electric bill.

The same work can be extended further by adding remote sensing technology like Bluetooth to control the system from a short-range and $\mathrm{Wi}-\mathrm{Fi}$ to control the system remotely from anywhere in the globe.

\section{Acknowledgment}

None.

\section{Conflicts of interest}

The authors have no conflicts of interest to declare.

\section{References}

[1] Abd Wahab MH. IoT-based home automation system for people with disabilities. In 5th international conference on reliability, infocom technologies and optimization (Trends and Future Directions) 2016 (pp. 51-1). IEEE.

[2] Jabbar WA, Alsibai MH, Amran NS, Mahayadin SK. Design and implementation of IoT-based automation system for smart home. In 2018 international symposium on networks, computers and communications 2018 (pp. 1-6). IEEE.

[3] Sulayman II, Almalki SH, Soliman MS, Dwairi MO. Designing and implementation of home automation system based on remote sensing technique with Arduino uno microcontroller. In IEEE-GCC conference and exhibition 2017 (pp. 1-9). IEEE.

[4] Gunputh S, Murdan AP, Oree V. Design and implementation of a low-cost Arduino-based smart home system. In 9th international conference on communication software and networks 2017 (pp. 1491-95). IEEE.
[5] Kumar N, Singh P. Economical home automation system using Arduino UNO. Advances in Computational Sciences and Technology. 2017; 10(6):1861-6.

[6] Nayyar C, Valarmathi B, Santhi K. Home security and energy efficient home automation system using Arduino. In international conference on communication and signal processing 2017 (pp. 1217-21). IEEE.

[7] Panigrahi A, Sahu B, Majhi AK, Bairganjan BC. Opportunities and challenges of blockchain in healthcare. International Journal of Control and Automation. 2020; 13(2):1731-7.

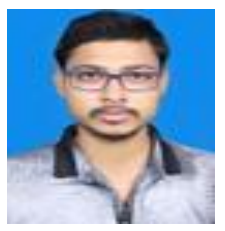

Asish Kumar Majhi is an Mphil Scholar in the Department of Computer Application Maharaja SriRam Chandra BhanjaDeo University (formerly known as North Orissa University), Takatpur, Baripada, Odisha, India.

Email: sinuashish@gmail.com

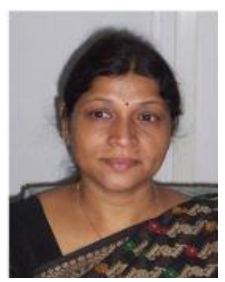

Sujata Dash is an Associate Professor in the Department of Computer Application Maharaja SriRam Chandra BhanjaDeo University (formerly known as North Orissa University), Takatpur, Baripada, Odisha, India

Email: sujata238dash@gmail.com

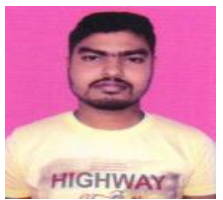

Chandan Kumar Barik is an Mphil Scholar in the Department of Computer Application Maharaja SriRam Chandra BhanjaDeo University (formerly known as North Orissa University), Takatpur, Baripada, Odisha, India

Email: chandankumarbarik886@gmail.com 